\title{
A Method for Solving Initial and Boundary Value Fuzzy differential equations by Mellin Transform
}

Noreen Azhar ( $\nabla$ noreen_nadeem@yahoo.com )

Sardar Bahadur Khan Women's University, Quetta https://orcid.org/0000-0002-3209-1301

\section{Saleem lqbal}

University of Balochistan

\section{Research Article}

Keywords: Fuzzy valued function, Fuzzy Mellin transform, fuzzy derivatives, Fuzzy differential equation, initial and boundary value conditions

Posted Date: March 9th, 2021

DOI: https://doi.org/10.21203/rs.3.rs-301071/v2

License: (c) (1) This work is licensed under a Creative Commons Attribution 4.0 International License.

Read Full License 


\section{(Arabian Journal for Science and Engineering)}

\section{A Method for Solving Initial and Boundary Value Fuzzy differential equations by Mellin Transform}

Noreen Azhar ${ }^{1}$, Saleem Iqbal ${ }^{2}$

${ }^{1}$ Mathematics Department, Sardar Bahadur Khan Women's University, Quetta, Pakistan, noreen_nadeem@yahoo.com

${ }^{2}$ Mathematics Department, University of Balochistan, Quetta, Pakistan, saleemiqbal81@yahoo.com 


\begin{abstract}
This Paper is included fuzzy concepts of Mellin transform along with its operational properties. Mellin transform method is applicable in fuzzy context. The study involved the proposed techniques for solving initial and boundary value fuzzy differential equations under strongly generalized differentiability concepts.
\end{abstract}

Keywords: Fuzzy valued function, Fuzzy Mellin transform, fuzzy derivatives, Fuzzy differential equation, initial and boundary value conditions.

\title{
Introduction
}

Various integral transforms are applicable in the field of engineering and mathematics. Mellin integral transform is undoubtedly one of them, which is used as a tool to explore new techniques of research in pure and applied mathematics. Differential equations extracted from many physical problems can also be solved by Mellin integral transform techniques. Any dynamical systems can be modeled under uncertainty and imprecision in a proper way by using Fuzzy differential equations [1]. Mellin integral equations can also be established as Fuzzy Mellin transform which is helpful in solving various physical problems. Under some strong differential conditions, Fuzzy Mellin transform is applicable for the solution of fuzzy differential equations [2].

Zadeh and Chang had initially gave the concept of fuzzy derivatives [3]. Later, extension principle were added to fuzzy derivatives theorem by Prade and Dubois [4]. Differentiability of fuzzy valued function discussed by several methods such as fuzzy-valuedness as a generalization of the Hukuhara derivatives and concept of Hukuhara difference of sets in fuzzy derivatives was introduced by Puri and Ralescu [5]. Bede extended the generalized differentiability in fuzzy valued function and also applied in fuzzy differential equations [6] [7]. Mosleh et al. approximated the system of fuzzy linear differential equations under generalized differentiability [8]. Furthermore, Chalco Cano et al. expressed the techniques and formulation to solve fuzzy differential equations by a differential inclusion with the help of many examples showing the corrected and incorrect procedure for solving differential equations [9]. Fuzzy differential equations can also be solved by using suitable numerical methods under generalized differentiability [10]. Various integral transforms also helpful for the solution of FDEs, in which fuzzy Laplace integral transform, Fuzzy Sumudu transform, Fuzzy Fourier transform and Fuzzy Mellin transform etc are included. The concept of fuzzy Laplace transform was given by Salahshour et al. in [11], moreover he solved FDEs by using this integral technique in [12]. In 2015, Khodadadi had attempt to solve fractional order fuzzy partial differential equations by fuzzy Fourier and fuzzy Laplace transforms [13]. Jafari and Sina in 2018 solved FDEs belonging to uncertain nonlinear system by using fuzzy Sumudu transform method [14].

Present research paper is comprises some basic concepts of fuzzy numbers, sets and fuzzy valued function which further used in defining fuzzy derivatives and integration, methodology is described by defining fuzzy Mellin transform and its some important properties. Method for the solution of fuzzy differential equations by applying fuzzy Mellin transform also proposed both for initial and boundary value fuzzy differential equations. Fuzzy Mellin transform and its operator properties are convenient to establish new results for solving FDEs. 


\section{Basic concepts}

This section will briefly present the basic concept of fuzzy numbers, it is denoted by a mapping $u: R \rightarrow[0,1]$, where $R$ is a set of all real numbers with the following properties: [15] , [16]

(a) $\mathrm{u}$ is upper semi-continuous,

(b) $\mathrm{u}$ is fuzzy convex, i.e., $u(\alpha x+(1-\alpha) y) \geq \min [u(x), u(y)] \forall x, y \in R$ and $u \in[0,1]$,

(c) $u$ is normal, i.e., $\exists x_{0} \in R$ for which $u\left(x_{0}\right)$,

(d) $\operatorname{supp} u=\{x \in R \mid u(x)>0\}$, its closure cl(supp u) is compact.

Let $B$ be the set of all fuzzy number on $R$. The a-level set of a fuzzy number $u \in B, 0 \leq a \leq 1$, denoted by $[u]_{a}$, is defined as

$$
[u]_{a}= \begin{cases}\{x \in R \mid u(x)>a\} & \text { if } 0 \leq a \leq 1 \\ \operatorname{cl}(\operatorname{supp~u}) & \text { if } \mathrm{a}=0\end{cases}
$$

Clearly the a-level set of a fuzzy number is a closed and have bounded interval $[u(a), \bar{u}(a)]$ also each $y \in R$ can be considered as a fuzzy numbers $\hat{y}$ defined by

$$
\hat{y}(b)= \begin{cases}1 & \text { if } b=y \\ 0 & \text { if } b \neq y\end{cases}
$$

Parametric form of Fuzzy number $u$ is in pair of function; $u(r)$ and $\bar{u}(r)$ where $0 \leq r \leq 1$, following conditions are satisfied under definition in which a functions $u(r)$ is bounded nondecreasing left continuous function in $(0,1]$, and right continuous at 0 and $\bar{u}(r)$ is bounded nonincreasing left continuous function in $(0,1]$, and right continuous at 0 , such that $u(r) \leq \bar{u}(r)$ where $0 \leq r \leq 1$. Under the same assumption fuzzy numbers also satisfy basic arithmetic operations of addition $u \oplus v$, subtraction $u \ominus v$, multiplication $u \odot v$ and scalar multiplications by $k$ i.e., $k \odot u$. More precisely, according to Zadeh's extension principle

$$
\begin{array}{r}
\left(u_{1}+u_{2}\right)\left(x_{0}\right)=\sup _{y \in R} \min \left\{u_{1}(y), u_{2}\left(x_{0}-y\right)\right\}, x \in R \\
(k u)\left(x_{0}\right)=u\left(x_{0} / k\right), k>0, \text { and }(k u)\left(x_{0}\right)=\tilde{0} \in B, k=0 .
\end{array}
$$

Now The Hausdroff distance between two fuzzy numbers $u_{1}$ and $u_{2}$ where $u_{1}, u_{2} \in B$ is defined by [17]

$D: B \times B \rightarrow R_{+} \cup 0$, where $D$ is metric in $B$

$$
D(u, v)=\sup _{r \in[0,1]} \max |u(r)-v(r)|,|u(r)-v(r)|,
$$

Here $u=(u(r), \bar{u}(r)), v=(v(r), \bar{v}(r)) \subset R$ which satisfy all the properties of a complete metric space $B$.

Since we know that $B$ is the set of all fuzzy number on $R$ then $f$ is a fuzzy valued function defined by $f: R \rightarrow B$

Differentiability of fuzzy mapping is defined in a sense of Hukuhara derivatives and is known as $\mathrm{H}$-derivative based on $\mathrm{H}$-difference of sets. It follows that Let $x, y \in B$. If there exists $z \in B$ 
such that $x=y \oplus z$, then $\mathrm{z}$ is called the H-difference of $x$ and $y$, and it is denoted by $x-{ }^{\hbar} y$. Here we denote $\mathrm{H}$-difference by the notation $-\hbar$.

Furthermore, Bede and Gal in 2005 defined the fuzzy differentials with four main conditions as Let $f:(a, b) \rightarrow B$ and $x_{0} \in(a, b)$, since $\mathrm{f}$ is strongly generalized differential at $x_{0}$ if $\exists$ an element $f^{\prime}\left(x_{0}\right) \in B$ such that [7]

1. $\forall$ sufficiently small $h, \exists f\left(x_{0}+h\right)-{ }^{\hbar} f\left(x_{0}\right), \exists f\left(x_{0}\right)-{ }^{\hbar} f\left(x_{0}-h\right)$ and having limits defined in metric D

$$
\lim _{h \searrow 0} \frac{f\left(x_{0}+h\right)-{ }^{\hbar} f\left(x_{0}\right),}{h}=\lim _{h \searrow 0} \frac{f\left(x_{0}\right)-{ }^{\hbar} f\left(x_{0}-h\right)}{h}=f^{\prime}\left(x_{0}\right)
$$

2. $\forall$ sufficiently small $h, \exists f\left(x_{0}\right)-{ }^{\hbar} f\left(x_{0}+h\right), \exists f\left(x_{0}-h\right)-{ }^{\hbar} f\left(x_{0}\right)$ and having limits defined in metric D

$$
\lim _{h \searrow 0} \frac{f\left(x_{0}\right)-{ }^{\hbar} f\left(x_{0}+h\right),}{-h}=\lim _{h \searrow 0} \frac{f\left(x_{0}-h\right)-{ }^{\hbar} f\left(x_{0}\right)}{-h}=f^{\prime}\left(x_{0}\right)
$$

3. $\forall$ sufficiently small $h, \exists f\left(x_{0}+h\right)-{ }^{\hbar} f\left(x_{0}\right), \exists f\left(x_{0}-h\right)-\hbar f\left(x_{0}\right)$ and having limits defined in metric D

$$
\lim _{h \unlhd 0} \frac{f\left(x_{0}+h\right)-{ }^{\hbar} f\left(x_{0}\right),}{h}=\lim _{h \unlhd 0} \frac{f\left(x_{0}-h\right)-{ }^{\hbar} f\left(x_{0}\right)}{-h}=f^{\prime}\left(x_{0}\right)
$$

4. $\forall$ sufficiently small $h, \exists f\left(x_{0}\right)-{ }^{\hbar} f\left(x_{0}+h\right), \exists f\left(x_{0}-h\right)-{ }^{\hbar} f\left(x_{0}\right)$ and having limits defined in metric D

$$
\lim _{h \searrow 0} \frac{f\left(x_{0}\right)-{ }^{\hbar} f\left(x_{0}+h\right),}{-h}=\lim _{h \searrow 0} \frac{f\left(x_{0}-h\right)-{ }^{\hbar} f\left(x_{0}\right)}{h}=f^{\prime}\left(x_{0}\right)
$$

Another definition by Chalco-Cano and Roman-Flores in 2006 for differential functions [18]

Let $F: R \rightarrow B$ be a fuzzy valued function and denote $F(t)=\left[f_{\alpha}(t) g_{\alpha}(t)\right]$, for each $\alpha \in\{0,1\}$ then

1. If $f$ is (i)- differentiable, then $f_{\alpha}(t)$ and $g_{\alpha}(t)$ are differentiable functions which are the lower and the upper functions of fuzzy-valued function $f$ in the parametric form and $F^{\prime}(t)=\left[f^{\prime}{ }_{\alpha}(t) g^{\prime}{ }_{\alpha}(t)\right]$

2. If $f$ is (ii)- differentiable, then $f_{\alpha}(t)$ and $g_{\alpha}(t)$ are differentiable functions and $F^{\prime}(t)=$ $\left[g_{\alpha}^{\prime}(t) f^{\prime}{ }_{\alpha}(t)\right]$

Fuzzy Riemann integrable function was introduced by Stefanini and Bede, according to them let $u(x, t)=(u(x, t), \bar{u}(x, t))$ be a fuzzy valued function on $[a, \infty]$, for every $b \geq a$ there exist two positive numbers; $M(t)$ and $\bar{M}(t)$ such that $\int_{a}^{b}|u(x, t)| d x \leq M(t)$ and $\int_{a}^{b}|\bar{u}(x, t)| d x \leq \bar{M}(t)$, then $u(x)$ is improper fuzzy Riemann-integrable on [a, $\infty$ ] [19]

and the improper integral for this is expressed as 


$$
I_{u}=\int_{a}^{\infty} u(x, t) d x=\left[\int_{a}^{\infty} u(x, t) d x, \int_{a}^{\infty} \bar{u}(x, t) d x\right.
$$

According to Tamir et al. fuzzy complex valued function has a membership function defined by an expression $z(t)=z_{1}(t)+i z_{2}(t)$, where $z_{1}, z_{2} \in R \rightarrow[0,1]$ and $t \in R$ and it can satisfy all the basic properties of fuzzy set that is $z$ is normal, semi-continuous and its $\alpha$-level set is always convex, compact. [20]

\section{Material and Methods.}

The concept of Fuzzy set theory and fuzzy numbers considered as an important tool for better understanding of some real situations in uncertain context.

\subsection{Fuzzy Differential equations.}

Fuzzy differential equation mainly used to provide information about uncertain dynamical system and give more flexible and realistic model. Involvement of fuzzy number make it different from ordinary differential equations.

Consider fuzzy differential equation with the Hukuhara fuzzy derivative and initial conditions i.e.,

$$
\dot{u}=f(t, u(t)), \quad u(0)=u_{0},
$$

Where $f:[0, T] \times B \rightarrow B$ is a fuzzy mapping, $u_{0}, \in B$ also a mapping defined $u:[0, T] \rightarrow B$ is called solution of fuzzy differential equation if it is absolutely continuous and satisfies it everywhere on $[0, T]$ by means of integral equation

$$
u(t)=u_{0}+\int_{t_{0}}^{t} f(s, u(s) d s, \quad t \in[0, T]
$$

also if $\mathrm{u}(\mathrm{t})$ satisfies the above integral equation then

$\operatorname{dia}\left[u(t)^{\alpha}\right] \geq \operatorname{dia}\left[u_{0}\right]^{\alpha}, \quad \alpha \in[0,1]$ where dia means diameter of the set involved.

When $u=u(t)$ is a fuzzy number, then according to Zadeh principle, it can be defined as

$$
(f(t, u(t)(s)=\sup \{u(\tau): s=f(t, \tau)\}, \quad s \in R
$$

It follows that

$$
\left[f(t, u(t)]^{\alpha}=\left[f_{1}^{\alpha}(t, u(t)), f_{2}^{\alpha}(t, u(t))\right], \quad \alpha \in[0,1]\right.
$$

Where

$$
\begin{gathered}
f_{1}^{\alpha}(t, u(t))=\min \left\{\mathrm{f}(\mathrm{t}, \mathrm{w}): \mathrm{w} \in\left[u_{1}^{\alpha}(t), u_{2}^{\alpha}(t)\right]\right\}, \quad 0<\alpha<1 \text { and } \\
f_{2}^{\alpha}(t, u(t))=\max \left\{\mathrm{f}(\mathrm{t}, \mathrm{w}): \mathrm{w} \in\left[u_{1}^{\alpha}(t), u_{2}^{\alpha}(t)\right]\right\}, \quad 0<\alpha<1
\end{gathered}
$$

Moreover Bede \& Gal given the existence of solutions of a fuzzy initial-value problem under generalized differentiability [7]

Fuzzy differential equations (FDE) can be solved by various integral transforms mainly Fourier, Laplace and Sumudu transforms. Salahshour in 2013 proposed two types of absolute values of 
fuzzy-valued functions also investigate the solutions of fuzzy initial value problems (FIVPs) and the solutions in state-space description of fuzzy linear continuous-time systems under generalized H-differentiability. [12]. Fuzzy boundary value problems under generalized differentiability solved by using Laplace transform proposed in [21] [12]. According to Xian Sun provided the solution of FDE under strongly generalized differentiability condition by using fuzzy Mellin transform. [2].

\subsection{Fuzzy Mellin Integral Transform.}

When we define the classical Mellin transform in fuzzy environment then it is called fuzzy Mellin Transform which can fulfilled all the requirement and conditions of complex fuzzy valued function.

$$
\mathcal{M}_{f}\{u(x), t\}=u(t)=\int_{0}^{\infty} x^{t-1} \odot u(x) d x \quad \text { where } t \in C
$$

Here $u(x)=u_{f}(x), v_{f}(x)$ is a continuous complex fuzzy-valued function.

Suppose $x^{t-1} \odot u_{f}(x)$ is improper fuzzy Riemann integrable on $(0, \infty)$

Then above becomes

$$
\begin{aligned}
\mathcal{M}_{f}\{u(x), t\}= & \left(\int_{0}^{\infty} x^{t-1} \odot u_{f}(x) d x, \int_{0}^{\infty} x^{t-1} \odot v_{f}(x) d x\right) \\
& =\mathcal{M}_{f}\left\{u_{f}(x)\right\}, \mathcal{M}_{f}\left\{v_{f}(t)\right\}
\end{aligned}
$$

The fuzzy Mellin transform converges absolutely and uniformly in the vertical strip $\delta_{-}<$ $\operatorname{Re}(t)<\delta_{+}$,

Where $\quad \delta-:=\inf \left\{\delta|| u(x) \mid=O\left(x^{-\delta}\right), x \rightarrow 0+\right\}$,

$$
\delta-:=\sup \left\{\delta|| u(x) \mid=O\left(x^{-\delta}\right), x \rightarrow \infty\right\},
$$

and for $\alpha<\operatorname{Re}(t)<\beta$, Mellin inverse transform defined as

$\mathcal{M}_{f}^{-1}\{u(t)\}=f(x)=\frac{1}{2 \pi i} \int_{b-i \infty}^{b+i \infty} \mathcal{M}_{f}[u(x), t] x^{-t} d t$, where $\delta_{-}<b<\delta_{+}$,

\section{Result and Discussion:}

Since Mellin transform describes operational methods to reduce the algebraic manipulations on transforms and is applied in area of mathematics and engineering, Now we are going to establish different properties of Mellin transform in a sense of fuzzy Mellin transform.

\subsection{Properties of Fuzzy Mellin Transform i. $\quad$ Scaling Property:}

Let $\mathcal{M}_{f}\{u(x), t\}=u(t)$, for $\alpha<\operatorname{Re}(t)<\beta$

$$
\mathcal{M}_{f}\{u(a x)\}=a^{-t} \odot u(t), \quad a>0 \& x>0
$$

Since $\mathcal{M}_{f}$ is a linear operator in the domain of the strips of convergence $\alpha<\operatorname{Re}(t)<\beta$ 


\section{ii. Shifting property:}

From above definition of FMT, we have the results

$$
\begin{aligned}
& \text { a) } \mathcal{M}_{f}\left\{u\left(x^{a} \odot u(x)\right\}=u(t \oplus a)\right. \\
& \text { b) } \mathcal{M}_{f}\left\{u\left(x^{a}\right)\right\}=\frac{1}{a} \odot u\left(\frac{t}{a}\right)
\end{aligned}
$$

By using these results we have following propositions with proof for the fuzzy Mellin transform

$$
\text { Proposition 1. } \quad \mathcal{M}_{f}\left\{\frac{1}{x} \odot u\left(\frac{1}{x}\right)\right\}=u(1 \ominus t)
$$

Proof: Since from definition,

$$
\begin{aligned}
& \mathcal{M}_{f}\{u(x), t\}=\int_{0}^{\infty} x^{t-1} \odot u(x) d x \text {, for } \alpha<\operatorname{Re}(t)<\beta \\
& \text { Consider } u\left(\frac{1}{x}\right) \text { then } \\
& \qquad \mathcal{M}_{f}\left\{u\left(\frac{1}{x}\right), t\right\}=\int_{0}^{\infty} x^{t-1} \odot u\left(\frac{1}{x}\right) d x
\end{aligned}
$$

Multiply by $\frac{1}{x}$ on both sides we get,

$$
\begin{aligned}
& \mathcal{M}_{f}\left\{\frac{1}{x} u\left(\frac{1}{x}\right), t\right\}=\int_{0}^{\infty} \frac{1}{x} x^{t-1} \odot u\left(\frac{1}{x}\right) d x \\
& \mathcal{M}_{f}\left\{\frac{1}{x} u\left(\frac{1}{x}\right), t\right\}=\int_{0}^{\infty} x^{t-2} \odot u\left(\frac{1}{x}\right) d x
\end{aligned}
$$

Put $\frac{1}{x}=p \Rightarrow x=\frac{1}{p}$

and $d x=-\frac{1}{p^{2}} d p$

$$
\begin{gathered}
=\int_{0}^{\infty}\left(\frac{1}{p}\right)^{t-2} \odot u(p)-\frac{1}{p^{2}} d p \\
=\int_{0}^{\infty} p^{-t} \odot u(p) d p \\
=\int_{0}^{\infty} p^{-t+1-1} \odot u(p) d p \\
=u(1-t), \quad \text { proved }
\end{gathered}
$$

Proposition 2. $\mathcal{M}_{f}\left\{(\log x)^{n} \odot u(x)\right\}=\frac{d^{n}}{d t^{n}} \mathcal{M}_{f}\{u(x), t\}, \quad n=1,2,3, \ldots$.

Proof: By definition,

$$
\mathcal{M}_{f}\{u(x), t\}=\int_{0}^{\infty} x^{t-1} \odot u(x) d x=u(t), \text { for } \alpha<\operatorname{Re}(t)<\beta
$$




$$
\mathcal{M}_{f}\{\log x \odot u(x), t\}=\int_{0}^{\infty} x^{t-1} \odot \log x \odot u(x) d x
$$

By using the result, we have

$$
\begin{gathered}
\frac{d}{d t}\left(x^{t-1}\right)=x^{t-1} \odot \log x \\
\mathcal{M}_{f}\{\log x \odot u(x), t\}=\int_{0}^{\infty} \frac{d}{d t}\left(x^{t-1}\right) \odot u(x) d x \\
=\frac{d}{d t} \int_{0}^{\infty}\left(x^{t-1}\right) \odot u(x) d x \\
=\frac{d}{d t} u(t)=\frac{d}{d t} \mathcal{M}_{f}\{u(x), t\},
\end{gathered}
$$

where $\operatorname{Re}(t) \in(\alpha, \beta)$ and $n \in N$

above is the case for $n=1$,

For $n=2$, we have

$$
\begin{gathered}
\mathcal{M}_{f}\left\{(\log x)^{2} \odot u(x), t\right\}=\frac{d^{2}}{d t^{2}} \int_{0}^{\infty}\left(x^{t-1}\right) \odot u(x) d x \\
=\frac{d^{2}}{d t^{2}} u(t)=\frac{d^{2}}{d t^{2}} \mathcal{M}_{f}\{u(x), t\}
\end{gathered}
$$

Similarly for generalized case we can write

$$
\mathcal{M}_{f}\left\{(\log x)^{n} \odot u(x)\right\}=\frac{d^{n}}{d t^{n}} \mathcal{M}_{f}\{u(x), t\}, \quad n=1,2,3, \ldots .
$$

\section{iii. Fuzzy Mellin transform of derivatives:}

$$
\mathcal{M}_{f}\left\{u^{\prime}(x)\right\}=-(t-1) \odot u(t-1)
$$

Provided $\left\{x^{t-1} \mathrm{u}(\mathrm{x})\right\}$ vanishes at $\mathrm{x} \rightarrow 0$, and $x \rightarrow \infty$ also

$$
\mathcal{M}_{f}\{u / /(x)\}=-(t-1) \odot(t-2) \odot u(t-2)
$$

Generally we have

$$
\mathcal{M}_{f}\left\{u^{n}(x)\right\}=(-1)^{n} \odot \frac{\Gamma(t)}{\Gamma(t-n)} \odot u(t-n)
$$

Provided that

$$
\text { vanishes at } x \rightarrow 0 \text {, for } n=0,1,2, \ldots,(n-1)
$$


If $\mathcal{M}_{f}\{u(x)\}=u(t)$, then

$$
\mathcal{M}_{f}\left[\left(x \frac{d}{d x}\right)^{2} \odot u(x)\right]=\mathcal{M}_{f}\left[x^{2} \odot u^{\prime /}(x)+x \odot u^{\prime}(x)\right]=(-1)^{2} \odot t^{2} \odot u(t)
$$

and in general,

$$
\mathcal{M}_{f}\left[\left(x \frac{d}{d x}\right)^{n} \odot u(x)\right]=(-1)^{n} t^{n} \odot u(t)
$$

v. Fuzzy Mellin transform of integrals

$$
\mathcal{M}_{f}\left\{\int_{0}^{x} u(p) d p\right\}=-\frac{1}{t} \odot u(t+1)
$$

More generally,

$$
\mathcal{M}_{f}\left\{I_{n} \odot u(x)\right\}=\mathcal{M}_{f}\left\{\int_{0}^{x} I_{n-1} \odot u(p) d p\right\}=(-1)^{n} \frac{\Gamma(t)}{\Gamma(t-n)} \odot u(t+n)
$$

where $I_{n} \mathrm{f}(\mathrm{x})$ is the $n$th repeated integral of $\mathrm{f}(\mathrm{x})$ defined by

$$
I_{n} \odot u(x)=\quad \int_{0}^{x} I_{n-1} \odot u(p) d p
$$

\section{vi. Convolution type theorems.}

Let consider two Fuzzy Mellin transforms, If $\mathcal{M}_{f}\{f(x)\}=f(t)$, and If $\mathcal{M}_{f}\{g(x)\}=g(t)$, then

The fuzzy Mellin convolution product, denoted by $f * g$, of a real-valued function $f(x)$ and a fuzzy-valued function $g(x)$, both function are defined on positive real axis such that $\alpha<\operatorname{Re}(t)<\beta$ then convolution theorem for fuzzy Mellin transform is defined by following expressions.

$$
\mathcal{M}_{f}[f(x) * g(x)]=\mathcal{M}_{f}\left[\int_{0}^{\infty} f(\xi) g\left(\frac{x}{\xi}\right) \frac{d \xi}{\xi}\right]=f(t) \odot g(t)
$$

and also

$$
\begin{aligned}
& \quad \mathcal{M}_{f}[f(x) o g(x)]=\mathcal{M}_{f}\left[\int_{0}^{\infty} f(x \xi) g(\xi) d \xi\right]=f(t) \odot g(1-t) \\
& \text { for } \alpha<\operatorname{Re}(t)<\beta
\end{aligned}
$$

\subsection{Construction of Solution of Fuzzy initial value problem FIVP by Fuzzy Mellin transform.}

Consider the fuzzy initial value problem 


$$
\begin{aligned}
\dot{u} & =f(x, u(x)), \\
u(0)=[\bar{u}(0, \alpha), \underline{u}(0, \alpha)], & 0<\alpha<1
\end{aligned}
$$

Where $f: R_{+} \times B \rightarrow B$ is a continuous fuzzy mapping. From fuzzy Mellin method we have :

$$
\mathcal{M}_{f}\{\dot{u}(x)\}=\mathcal{M}_{f}\{f(x, u(x))\}
$$

Then we have following possibilities for solving above FIVP

Case I: If we take $\dot{u}(x)$ is (i) differentiable then, from above we have $\dot{u}(x)=\overline{\dot{u}}(x, \alpha), \underline{\dot{u}}(x, \alpha)$ and

$$
\mathcal{M}_{f}\{\dot{u}(x)\}=-(t-1) \odot u(t-1)
$$

i.e., the $1^{\text {st }}$ derivative of Mellin transform

It can be written as follows,

$$
\left\{\begin{array}{l}
m\{\bar{f}(x, u(x), \alpha)\}=-(t-1) \odot \bar{u}(t-1) \\
m\{\underline{f}(x, u(x), \alpha)\}=-(t-1) \odot \underline{u}(t-1)
\end{array}\right.
$$

Where $\bar{f}(x, u(x), \alpha)=\min \{f(x, w) \mid w \in(\bar{u}(x, \alpha), \underline{u}(x, \alpha)\}$ and

$$
\underline{f}(x, u(x), \alpha)=\max \{f(x, w) \mid w \in(\bar{u}(x, \alpha), \underline{u}(x, \alpha)\}
$$

We can assume for the sake of simplicity to solve the linear system:

$$
\begin{gathered}
m\{\bar{f}(x, \alpha)\}=K_{1}(t, \alpha) \\
m\{f(x, \alpha)\}=H_{1}(t, \alpha)
\end{gathered}
$$

Where $K_{1}(t, \alpha)$ and $H_{1}(t, \alpha)$ are the solution of the system (5.4). Now $\bar{u}(x, \alpha)$ and $\underline{u}(x, \alpha)$ can be computed by using inverse Mellin transform as follows:

$$
\begin{gathered}
\bar{f}(x, \alpha)=m^{-1}\left(K_{1}(t, \alpha)\right) \\
\underline{f}(x, \alpha)=m^{-1}\left(H_{1}(t, \alpha)\right)
\end{gathered}
$$

Case II: If we take $\dot{u}(x)$ is (ii) differentiable then, from above we have $\dot{u}(x)=$ $\dot{u}(x, \alpha), \bar{u}((x, \alpha)$ and

$$
\mathcal{M}_{f}\{\dot{u}(x)\}=-(t-1) \odot u(t-1)
$$

i.e., the $1^{\text {st }}$ derivative of Mellin transform

It can be written as follows, 


$$
\left\{\begin{array}{l}
m\{f(x, u(x), \alpha)\}=-(t-1) \odot \underline{u}(t-1) \\
m\{\bar{f}(x, u(x), \alpha)\}=-(t-1) \odot \bar{u}(t-1)
\end{array}\right.
$$

Where $f(x, u(x), \alpha)=\min \{f(x, w) \mid w \in(\underline{u}(x, \alpha), \bar{u}(x, \alpha)\}$ and

$$
\bar{f}(x, u(x), \alpha)=\max \{f(x, w) \mid w \in(\underline{u}(x, \alpha), \bar{u}(x, \alpha)\}
$$

By assuming solution of the linear system, we have

$$
\begin{aligned}
& m\{\bar{f}(x, \alpha)\}=K_{2}(t, \alpha) \\
& m \overline{\{f}(x, \alpha)\}=H_{2}(t, \alpha)
\end{aligned}
$$

Where $K_{2}(t, \alpha)$ and $H_{2}(t, \alpha)$ are the solution of the system (5.9). $\bar{u}(x, \alpha)$ and $\underline{u}(x, \alpha)$ can be computed by using inverse Mellin transform as follows:

$$
\begin{aligned}
& f(x, \alpha)=m^{-1}\left(K_{2}(t, \alpha)\right) \\
& \bar{f}(x, \alpha)=m^{-1}\left(H_{2}(t, \alpha)\right)
\end{aligned}
$$

We can follow the same assumptions for $2^{\text {nd }}$ order fuzzy differential equations.

$y^{\prime \prime}+a y^{\prime}+b y=\alpha(t) ; y\left(t_{0}\right)=k_{0}$ and $y^{\prime}\left(t_{0}\right)=k_{1}$

Where $a \& b$ are constants.

Here some differential equations are solved by using fuzzy Mellin transforms.

\subsection{Application.}

Example 1. Consider fuzzy Euler's nonhomogeneous differential equation.

$$
\left\{x^{2} \odot D^{2}+2 \odot x \odot D-2\right\} Y(x)=u \odot\left(x^{-3} H(a-x)\right) \quad a>0, u \in B
$$

Where $H(a-x)$ is Heaviside step function.

Solution: Applying Fuzzy Mellin transform on both sides of above equation, we get

$$
\begin{gathered}
\mathcal{M}_{f}\left\{x^{2} \odot D^{2}+2 \odot x \odot D-2\right\} Y(x)=\mathcal{M}_{f}\left\{u \odot\left(x^{-3} H(a-x)\right)\right\} \\
\left(t^{2}-t-2\right) Y(t)=u \odot \frac{a^{t-3}}{(t-3)} \quad a>0, \operatorname{Re}(t-3)>0 \\
Y(t)=u \odot \frac{a^{t-3}}{(t+1)(t-2)(t-3)}=u \odot \frac{a^{t-3}}{4(t-3)}-\frac{a^{t-2}}{3 a(t-2)}+\frac{a^{t+1}}{12 a^{4}(t+1)}
\end{gathered}
$$

Now inverse Mellin transform for $\operatorname{Re}(t)>3$, we have

$$
Y(x)=u \odot H(a-x) \odot\left(\frac{1}{4 x^{3}}-\frac{1}{3 a x^{2}}+\frac{x}{12 a^{4}}\right)
$$


Example 2: Consider the initial value problem equation,

$$
x^{2} \frac{d^{2} y}{d x^{2}}+4 y=\sigma \odot e^{-a x} ; \quad y^{\prime}(0)=0 \text { and } y(0)=\rho
$$

Where $\sigma=(3.2+0.8 \alpha, 5-\alpha)$ and $\rho=(0.8+0.2 \alpha, 1.5-0.5 \alpha)$ where $0<\alpha<1$

Solution: By using fuzzy Mellin transform method we have

$$
\mathcal{M}_{f}\left\{x^{2} \frac{d^{2} y}{d x^{2}}+4 y\right\}=\mathcal{M}_{f}\left\{\sigma \odot e^{-a x}\right\}
$$

In (ii) differentiable, we obtain $(-1)^{n} t^{2} f(t)$

$$
\begin{aligned}
& (-1)^{2} \odot t^{2} \odot \underline{y}(t, \alpha)+4 \underline{y}(t, \alpha)=\mathcal{M}_{f}\left\{5-\alpha \odot e^{-a x}\right\} \\
& (-1)^{2} \odot t^{2} \odot \bar{y}(t, \alpha)+4 \bar{y}(t, \alpha)=\mathcal{M}_{f}\left\{3.2+0.8 \alpha \odot e^{-a x}\right\}
\end{aligned}
$$

Hence the solution is obtained by solving equation

$$
\underline{y}(t, \alpha)=5-\alpha \odot \frac{\Gamma(t)}{a^{t}\left(t^{2}+4\right)}
$$

And

$$
\bar{y}(t, \alpha)=3.2+0.8 \alpha \odot \frac{\Gamma(t)}{a^{t}\left(t^{2}+4\right)}
$$

Example 3: Consider the fuzzy partial differential equation with non-constant coefficient

$$
x^{2} \odot \frac{\partial^{2} u}{\partial x^{2}}-2 x y \odot \frac{\partial^{2} u}{\partial x \partial y}+y^{2} \odot \frac{\partial^{2} u}{\partial y^{2}}=c \odot e^{-(x+y)} \quad \text { where } c \in B
$$

\section{Solution:}

Above equation can be solved by applying fuzzy multiple Mellin transform defined as

$$
\mathcal{M}_{x y}[u(x, y) ; p, q]=U(p, q)=\int_{0}^{\infty} \int_{0}^{\infty} u(x, y) x^{p-1} y^{q-1} d x d y
$$

where $\mathrm{p}$ and $\mathrm{q}$ are complex numbers, the notation $\mathcal{M}_{x y}[u(x, y) ; p, q]$ is used in order to show multiple Mellin transform with respect to $\mathrm{x}$ and $\mathrm{y}$.

So that by applying double fuzzy Mellin transform on both sides of equation, we get

$$
\mathcal{M}_{x y}\left[x^{2} \odot \frac{\partial^{2} u}{\partial x^{2}}-2 x y \odot \frac{\partial^{2} u}{\partial x \partial y}+y^{2} \odot \frac{\partial^{2} u}{\partial y^{2}}\right]=c \odot \mathcal{M}_{x y}\left[e^{-(x+y)}\right]
$$

Where $c$ is a fuzzy number and $c \in B$. 
From we have

$$
\mathcal{M}_{x y}\left\{x^{2} \frac{\partial^{2} u(x, y)}{\partial x^{2}} ; p, q\right\}=(-p)^{2} \odot U(p, q)
$$

Where $\sigma_{1}<\operatorname{Req}<\sigma_{2}, \alpha_{1}<\operatorname{Rep}<\alpha_{2}$

$$
\begin{gathered}
\mathcal{M}_{x y}\left\{\frac{\partial^{2} u(x, y)}{\partial y^{2}} ; p, q\right\}=(-q)^{2} \odot U(p, q) \\
\mathcal{M}_{x y}\left\{x y \frac{\partial^{2} u(x, y)}{\partial x \partial y} ; p, q\right\}=p q \odot U(p, q)
\end{gathered}
$$

Put in above, we obtain

$$
(p)^{2} \odot U(p, q)-2 p q \odot U(p, q)+(q)^{2} \odot U(p, q)=c \odot \Gamma(\mathrm{p}) \Gamma(\mathrm{q})
$$

And

$$
U(p, q)=\frac{c \odot \Gamma(\mathrm{p}) \Gamma(\mathrm{q})}{(p-q)^{2}}
$$

Inverse Mellin transform of the above function for $(x, y)$ ranging from- $\infty$ to $\infty$.

$$
U(x, y)=c \odot \frac{1}{4 \pi^{2}} \int_{-\infty}^{\infty} \int_{-\infty}^{\infty} \frac{\Gamma(p) \Gamma(q)}{(p-q)^{2}} x^{-p} y^{-q} d p d q \quad \text { where } p \neq q
$$

\section{Construction of Solution of Fuzzy Boundary value problem (FBVP) by Fuzzy Mellin transform}

Consider the boundary value condition for 2 nd order fuzzy differential equation

$$
\mathrm{u}^{\prime \prime}(\mathrm{x})=f\left(\mathrm{x}, \mathrm{u}(\mathrm{x}), \mathrm{u}^{\prime}(\mathrm{x})\right)
$$

subject to two-point boundary conditions

$$
\begin{aligned}
& \mathrm{u}(0)=(\mathrm{u}(0 ; \alpha), \mathrm{u}(0 ; \alpha)), \\
& \mathrm{u}(c)=(\mathrm{u}(c ; \alpha), \mathrm{u}(c ; \alpha)) \quad 0<\alpha<1
\end{aligned}
$$

Where $f: R_{+} \times B \rightarrow B$ is a continuous fuzzy mapping. From fuzzy Mellin method we have :

$$
\mathcal{M}_{f}\left\{\mathrm{u}^{\prime \prime}(\mathrm{x})\right\}=\mathcal{M}_{f}\left\{f\left(\mathrm{x}, \mathrm{u}(\mathrm{x}), \mathrm{u}^{\prime}(\mathrm{x})\right)\right\}
$$

Which can be written as

$$
\begin{aligned}
& \mathcal{M} \mathcal{M}_{f}\left\{\mathrm{u}^{\prime \prime}(\mathrm{x})\right\}=-(t-1) \odot(t-2) \odot u(t-2) \quad \text { (5.3)i.e; the 2nd derivative of Mellin } \\
& \text { transform }
\end{aligned}
$$

Then the classical form of fuzzy Mellin transform,

$$
m \bar{f}\left(\mathrm{x}, \mathrm{u}(\mathrm{x}), \mathrm{u}^{\prime}(\mathrm{x}), \alpha\right)=-(t-1) \odot \underline{u}(t-1, \alpha)
$$




$$
m\left\{\bar{f}\left(\mathrm{x}, \mathrm{u}(\mathrm{x}), \mathrm{u}^{\prime}(\mathrm{x}), \alpha\right)\right\}=-(t-1) \odot(t-2) \odot \bar{u}(t-1, \alpha)
$$

Then the classical form of fuzzy Mellin transform

Where $\underline{f}\left(\mathrm{x}, \mathrm{u}(\mathrm{x}), \mathrm{u}^{\prime}(\mathrm{x}), \alpha\right)=\min \{f(x, p) \mid p \in(\underline{u}(x, \alpha), \bar{u}(x, \alpha)\}$ and

$\bar{f}\left(\mathrm{x}, \mathrm{u}(\mathrm{x}), \mathrm{u}^{\prime}(\mathrm{x}), \alpha\right)=\max \{f(x, p) \mid p \in(\bar{u}(x, \alpha), \underline{u}(x, \alpha)\}$

we assume that $A(p ; \alpha)$ and $B(p ; \alpha)$ are the solutions of equations (6.4) and (6.5), then the above system becomes

$$
\begin{gathered}
m\{\underline{f}(x, \alpha)\}=A(p ; \alpha) \\
m\{\bar{f}(x, \alpha)\}=B(p ; \alpha)
\end{gathered}
$$

We get the upper and lower solution for given problem by using inverse Mellin transform as

$$
\begin{aligned}
& f(x, \alpha)=m^{-1}(A(p ; \alpha)) \\
& \bar{f}(x, \alpha)=m^{-1}(B(p ; \alpha))
\end{aligned}
$$

\section{Example 5.1.}

Consider the following fuzzy homogenous boundary value problem

$$
\mathrm{u}^{\prime \prime}(\mathrm{x})-3 \mathrm{u}^{\prime}(\mathrm{x})+2 \mathrm{u}(\mathrm{x})=0,
$$

subject to the following boundary conditions

$$
\begin{aligned}
& u(0)=(0.5 \alpha-0.5,1-\alpha), \\
& u(1)=(\alpha-1,1-\alpha) . \quad 0<\alpha<1
\end{aligned}
$$

Solution: By applying fuzzy Mellin transform on equation (6.11), we have

$$
\begin{array}{r}
\mathcal{M}_{f}\left\{\mathrm{u}^{\prime \prime}(\mathrm{x})-3 \mathrm{u}^{\prime}(\mathrm{x})+2 \mathrm{u}(\mathrm{x})\right\}=0, \\
\mathcal{M}_{f}\left\{\mathrm{u}^{\prime \prime}(\mathrm{x})\right\}=3 \mathcal{M}_{f}\left\{\mathrm{u}^{\prime}(\mathrm{x})\right\}-2 \mathcal{M}_{f}\{u(x)\}
\end{array}
$$

Since we know that

$$
\begin{gathered}
\mathcal{M}_{f}\left\{\mathrm{u}^{\prime \prime}(\mathrm{x})\right\}=-(t-1) \odot(t-2) \odot u(t-2) \text { and } \\
\mathcal{M}_{f}\left\{\mathrm{u}^{\prime}(\mathrm{x})\right\}=-(t-1) \odot u(t-1)
\end{gathered}
$$

Put in equation (6.12) we get,

$$
-(t-1) \odot(t-2) \odot u(t-2)=-3(t-1) \odot u(t-1)-2 u(t)
$$

Which is the difference equation, whose solution is 


$$
u(t)=\frac{1}{\left[\frac{(1-t)(t-2)}{x^{2}}+\frac{3(t-1)}{x}+2\right]}
$$

Now applying boundary conditions using the generalized differentiability case I, we have

$$
\begin{aligned}
u(0) & =0.5 \alpha-0.5=\frac{1}{2}, \text { and } \\
u(1)=\alpha-1 & =\frac{1}{(1-t)(t-2)+3(t-1)+2}
\end{aligned}
$$

now for using the generalized differentiability case II, we get

$$
\begin{gathered}
u(0)=1-\alpha=\frac{1}{2} \\
u(1)=1-\alpha=\frac{1}{(1-t)(t-2)+3(t-1)+2}
\end{gathered}
$$

The inverse Mellin transform of equation (6.14) can be find by using theory of residue on strips of convergence.

\section{Conclusion}

In this paper fuzzy Mellin transform along with its properties was interpreted to solve fuzzy differential equations by taking the concept of Hukuhara derivatives. Structure of finding initial and boundary value fuzzy differential equation by using Mellin transform is established. The method was illustrated by some examples and found to be accurate.

\section{Competing interests}

The authors declare that they have no competing interests.

\section{Author's Contributions}

The authors have contributed equally to the writing of this paper. They read and approved the manuscript.

\section{Acknowledgements.}

The authors are grateful to the learned referee for a very careful reading of the manuscript with many useful suggestions for improvement.

\section{Availability of data and materials.}


The sharing of data does not apply to this article since no data sets were produced or analyzed during the current study.

\section{References}

[1] M. Ghanbari, "Solution of the first order linear fuzzy differential equations by some reliable methods," Journal of Set Valued Analysis, vol. 2012, 2012.

[2] X. Sun and Z. yang, "A Mellin transform method for solving Fuzzy differential equations," Advances in Difference Equations, pp. 1-12, 2016.

[3] L. Zadeh and S. L. Chang, "On Fuzzy mapping and control," IEEE Trans. Systems Man Cybernet, vol. 2, pp. 30-34, 1972.

[4] D. Dubois and H. Prade, "Towards Fuzzy Differential Calculus," Fuzzy Sets and System, vol. 8, pp. 17, 1982.

[5] M. L. Puri and D. A. Ralescu, "Differentials for fuzzy function," Journal of Mathmaatical Analysis and Applicaaations, vol. 91, pp. 552-558, 1983.

[6] B. Bede, I. Rudas and A. Bencsik, "First order linear fuzzy differential equationsunder generalized differentiability," Information Sciences, vol. 177, pp. 1648-1662, 2007.

[7] B. Bede and S. G. Gal, "Generalizations of the differentiability of fuzzy-number-valued functions with applications to fuzzy differential equations," Fuzzy Sets and Systems, vol. 151, pp. 581-599, 2005.

[8] M. Mosleh and M. Otadi, "Approximate solution of fuzzy differential equations under generalized differentiability," Applied Mathmatical Model, vol. 39, pp. 3003-3015, 2015.

[9] Y. Chalco-Cano and H. Román-Flores, "Some remarks on fuzzy differential equations via differential inclusions," Fuzzy Sets and System, vol. 230, pp. 3-20, 2013.

[10] J. J. Nieto, A. Khastan and K. Iyaz, "Numerical solution of fuzzy differential equations under generalized differentiability," Nonlinear Analysis: Hybrid Systems, vol. 3, no. 4, pp. 700-707, 2009.

[11] S. Salahshour, T. Allahviranloo and S. Abbasbandy, "Solving fuzzy fractional differential equations by fuzzy Laplace transforms," Communications in Nonlinear Science and Numerical Simulation, vol. 17, pp. 1372-1381, 2012.

[12] S. Salahshour and T. Allahviranioo, "Applications of Fuzzy Laplace transforms," Soft Computing, vol. 17, pp. 145-158, 2013.

[13] E. Khodadadi and M. Karabacak, "Solving fuzzy fractional partial differential equations by fuzzy Laplace-Fourier transforms," Journal of Computational Analysis and Applications, vol. 19, no. 2, pp. 260-271, August 2015. 
[14] R. Jafri and S. Razvarz, "Solution of Fuzzy Differential Equations Using Fuzzy Sumudu Transforms," Mathematical and Computational Applications, vol. 23, no. 5, pp. 2-15, 2018.

[15] M. Friedman, M. Ma and K. Abraham, "Numerical solution of fuzzy differential and integral equations," Fuzzy Sets and system, no. 106, pp. 35-48, 1999.

[16] S. Song and C. Wu, "Existance and uniqueness of solutions to Cauchy problem of fuzzy differential equations," Fuzzy Sets and System, vol. 110, pp. 55-67, 2000.

[17] M. Puri and D. Ralescu, "Puri, M.L.; Ralescu, D. Fuzzy random variables," Journal of Mathematical Analysis and Applications, pp. 409-422, 1986.

[18] Y. Chalco-Cano and H. Roman-Flores, "Chalco-Cano Y, On new solutions of fuzzy differential equations.," Chaos Solitons Fractals, vol. 38, pp. 112-119, 2006.

[19] L. Stefanini and B. Bede, "Generalized Hukuhara differentiability of interval-valued functions and interval differential equations," Nonlinear Anal., Theory Methods and Applications, vol. 71, pp. 1311-1328, 2009.

[20] D. Tamir, L. Jin and A. Kandel, "a new interpretation of complex membership grade," journal of Intell. Fuzzy System, pp. 285-312, 2011.

[21] A. Khastan and J. J. Nieto, "Khastan, A , and Nieto, JJ, A boundary value problem for second order fuzzy differential equations," Nonlinear Analysis: Theory, Methods \& Application, vol. 72, no. 9, pp. 3583-3593, 2010. 
